\title{
Numerical Modeling of Torch Design for Plasma Atomization
}

\begin{abstract}
E. YurtKuran*, R. Unal and A. Ozsunar
Gazi University Faculty of Engineering Department of Mechanical Engineering, 06570 Ankara, Turkey

In this study, internal geometry of a non-transferred plasma torch was investigated for plasma atomization. Numerical analysis was performed by a computational fluid dynamics approach for the plasma jet formation. Gas velocity and temperature distribution at the plasma jet formed outside of the torch is very important for plasma atomization. In terms of achieving high jet temperature and jet velocity the internal geometry is the most important factor that affects the efficiency of the atomization. In a high-efficiency plasma torch, the gas velocity and temperature must be as high as possible at the plasma jet. For this reason, the effect of gas flow rate and current value on the gas velocity and temperature in the plasma jet is investigated parametrically. Numerical solutions of the calculated fluid dynamics are given and analysis results are given to determine the most suitable working conditions. The results showed that the internal geometry has significant effect on the plasma jet temperature and velocity at the outside of the torch.
\end{abstract}

DOI: 10.12693/APhysPolA.134.292

PACS/topics: plasma atomization, torch, CFD

\section{Introduction}

Plasma atomization process is developing very fast nowadays for the new generation of 3D printing manufacturing method. Today, Ti and alloy powders are used and their use is increasing rapidly for 3D printing process. The most important equipment for plasma atomization is the plasma torch. Plasma jet is produced in nozzle section of the torch. For the metal powder production with plasma atomization process, it is necessary to know the structure and characteristics of the plasma jet. The response of the plasma jet in terms of gas velocity, jet shape, jet length, and gas density is important according to the input energy. In the literature, there is no complete answer to the plasma jet behavior for plasma atomization process. Actually, the plasma jet properties mainly depend on the geometry of the nozzle/torch design. For that reason, the nozzle/torch design is very important. Experimental work in this area is very limited due to the high cost and difficulty of obtaining the data in the torch. Numerical simulation studies are performed as the most effective way to determine the physical and thermal conditions in the torch instead of the experimental ones. When the literature is examined, it is seen that plasma torch studies focus especially on the inner part. Many applications of electric arcs have been carried out, especially with non-transferred arc, in order to provide high quality and assist in the development of technology in special applications [1]. These studies are divided into two groups as $2 \mathrm{D}$ and $3 \mathrm{D}$.

For 2D models, the anode arc attachment is calculated axisymmetric [2]. Axial symmetric assumption is the most serious constraint away from reality when considering the anode arc connection, which simply eliminates

*corresponding author; e-mail: yurtkuranemre@gazi.edu.tr flow conditions [3]. This approach results in an unrealistic consequence [4]. In a study comparing $2 \mathrm{D}$ and $3 \mathrm{D}$ models, temperature and velocity profiles at the torch outlet were not symmetric [5]. For simulating arc behavior between the anode and the cathode, it is generally assumed that the main body of the arc is in the state of local thermodynamic equilibrium (LTE), and different physical/mathematical models are constructed by solving the combined orbit equations in these two sub-regions [6]. Another common assumption is that the plasma is optically thin and radiation is considered as a volumetric loss from plasma [7].

3D models are collected in two groups, time dependent and steady. In the vast majority of these studies, the position arc-root attachment in a DC plasma torch was obtained based on the Steenbeck principle $[3,6]$ or the minimum entropy principle [8]. The Steenbeck principle is a special case of the minimum entropy production principle. In addition, Eichert et al. [9] and Meillot et al. [10] used the thermal efficiency criterion to determine the length of arc-root attachment. Selvan et al. [11] estimated the different arc sizes using the thermodynamic principles of minimum entropy production to give torch power, and indicated that the nozzle output had a stronger three-dimensional effect. Trelles [12] simulated the plasma flow in a DC torch assuming that there is a layer of high electrical conductivity in front of the anode wall. Using this technique, the position of the arc-root attachment can also be estimated. In the paper of Guo et al. [4] the minimum total heat transfer rate along the anode wall, a new method for determining the location of the arc-arch attachment, is considered the criterion for the lowest energy loss. Based on this criterion, the actual arc core radius and length are estimated. Transient studies of Park et al. [7] modeled the motion of the arc that is guided by a rotating magnetic field. They aimed at reducing the wear of the anode by driving the magnetic field and distributing it rather than the single point. Experimental studies [11, 13] on plasma torches 
have shown that erosion caused by the arc-root attachment is at a fixed point. Therefore, steady numerical studies are also necessary and feasible [4].

In this study, in addition to the existing torch studies, an arc plasma torch geometry created Ar direct current (DC) non-transferred arc for plasma atomization method. The most important parameters for plasma atomization are the temperature and velocity outside the torch, because temperature and velocity are necessary to melt the solid wire and to disintegrate the melt for the droplet formation. As it is understood from commercial studies, more than one torch is used in this method. These torches are positioned at different angles. There is a distance between the wire and the torch exit. Therefore, these values should be transferred away from the torch outlet without falling off the energies.

\section{Theoretical method}

\subsection{Model assumptions}

In the literature on plasma formation, the following assumptions were made.

- The gravitational force and viscous dissipation is neglected as it is very small compared to the kinetic effects $[4,5,14]$.

- Plasma is in local thermodynamic equilibrium (LTE) assumed to simplify the mathematical formulae. As a result, the plasma gas is considered as continuous fluid characterized by a single temperature $[3,4]$.

- Based on the LTE assumption, the thermodynamic and transport properties of the plasma gas (such as $c_{p}, \mu$, and $\lambda$ ) are determined by the gas temperature, excluding the electrical conductivity $(\sigma)[14]$.

- Plasma flow is considered stable, turbulent and incompressible. Rapid warm-up causes sudden expansion around the arc, resulting in rapid acceleration, resulting in turbulent flow [3].

- The power supply is stable with a constant power rating. The induced electric field is insignificantly compared to the electric field intensity applied in the plasma arc region.

- The plasma is optically thin and radiation events are not considered.

\subsection{Governing equations}

Equations used in plasma jet modeling include equations of mass, momentum, and energy conservation. The equations based on the modeling of the arc part critical to plasma generation are the electromagnetic equations given by Maxwell's equations in the form of electric potential and magnetic vector potential. The related
Maxwell equations are Eqs. (1)-(7) and the other electromagnetic (the Ohm law) equations are Eqs. (8)-(10).

$$
\begin{aligned}
& \nabla \cdot \rho \boldsymbol{v}=0, \\
& \nabla \cdot(\rho \boldsymbol{v} \boldsymbol{v})=-\nabla p+\nabla \cdot \stackrel{\leftrightarrow}{\tau}+\boldsymbol{j} \times \boldsymbol{B}, \\
& \nabla \cdot(-\sigma \nabla \Phi)=0, \\
& \boldsymbol{E}=-\nabla \Phi, \\
& \Delta \boldsymbol{A}=-\mu_{0} \boldsymbol{J}, \\
& \boldsymbol{B}=\nabla \times \boldsymbol{A}, \\
& \nabla \cdot \boldsymbol{j}=0, \\
& \boldsymbol{J}=\sigma \boldsymbol{E}, \\
& \boldsymbol{j}=\sigma(-\nabla \Phi+\boldsymbol{v} \times \boldsymbol{B}) \\
& \nabla \cdot(\sigma \nabla \Phi)=\nabla \cdot(\sigma \boldsymbol{v} \times \boldsymbol{B}),
\end{aligned}
$$

where the term $\boldsymbol{j} \times \boldsymbol{B}$ - the Lorentz force, $\rho$ - density $\left[\mathrm{kg} / \mathrm{m}^{3}\right], \boldsymbol{v}$ - velocity $[\mathrm{m} / \mathrm{s}], \boldsymbol{j}$ - current density $\left[\mathrm{A} / \mathrm{m}^{2}\right], \boldsymbol{B}$ - magnetic field $[\mathrm{T}], \overleftrightarrow{\tau}$ - stress tensor [Pa], $\sigma$ - electrical conductivity $[1 /(\Omega \mathrm{m})], \boldsymbol{E}$ - electrical field $[\mathrm{V} / \mathrm{m}], \Phi$ - electrical potential $[\mathrm{V}]$. The sudden heating of the gas conveyed into the plasma nozzle, due to the arc, and the sudden acceleration due to the expansion around the arc, makes the flow turbulent. The $k-\varepsilon$ turbulence model was used to reach reliable solution and to model the turbulent flow. The kinetic energy of the turbulence $k$ Eq. (11) and the turbulence emission rate $(\varepsilon)$ from the model mathematical equations are given in Eq. (12):

$$
\begin{aligned}
& \nabla \cdot(\rho k v)=\nabla \cdot\left[\left(\mu+\frac{\mu_{t}}{\sigma_{k}}\right) \nabla k\right]+G_{k}-\rho \varepsilon, \\
& \nabla \cdot(\rho \varepsilon v)=\nabla \cdot\left[\left(\mu+\frac{\mu_{t}}{\sigma_{\varepsilon}}\right) \nabla \varepsilon\right]+\frac{\varepsilon}{k}\left(C_{1 \varepsilon} G_{k}-\rho \varepsilon C_{2 \varepsilon}\right),
\end{aligned}
$$

where the term $k$ - turbulent kinetic energy, $\varepsilon$ - turbulent dissipation rate, $\mu-$ dynamic viscosity $\left[\mathrm{N} \mathrm{s} / \mathrm{m}^{2}\right], \mu_{t}$ - turbulent viscosity $\left[\mathrm{N} \mathrm{s} / \mathrm{m}^{2}\right], G_{k}$ represents the generation of turbulence kinetic energy due to the mean velocity gradients. $\sigma_{k}$ and $\sigma_{\varepsilon}$ are the turbulent Prandtl numbers for $k$ and $e$, respectively. $C_{1 \varepsilon}$ and $C_{2 \varepsilon}$ are constant.

\subsection{Computational domain and boundary conditions}

Figure 1 shows the computational domain used to simulate the plasma jet. It is discretized into 799252 elements. Mesh is refined at cathode tip region. The boundary conditions for the calculation area are shown in Table I, where the term SLPM is standard litre per minute. 
The cathode temperature was set at $3500 \mathrm{~K}$ (Table I) in order to avoid divergence as the temperature rises suddenly. In this study the valid solution method is used $k-\varepsilon$ turbulence model.

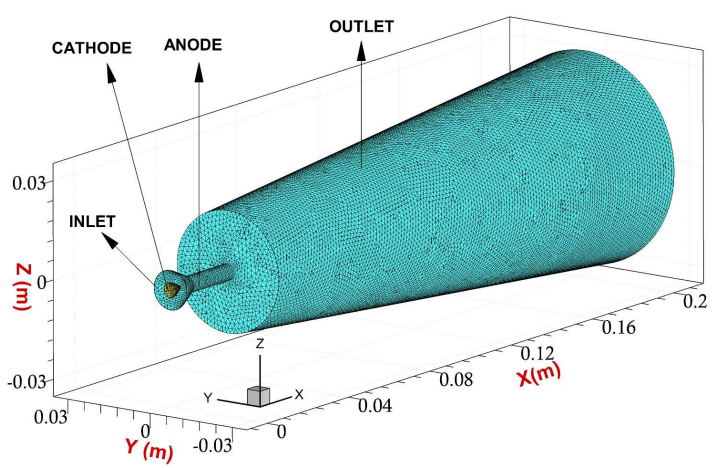

Fig. 1. Geometry of the computational domain and mesh.

Boundary conditions of the model used

TABLE I in the simulation.

\begin{tabular}{c|c|c|c|c}
\hline \hline & Inlet & Cathode & Anode & Outlet \\
\hline$P$ & 3 bar & $\partial P / \partial n=0$ & $\partial P / \partial n=0$ & 101325 \\
\hline$T$ & $300 \mathrm{~K}$ & $3500 \mathrm{~K}$ & $1000 \mathrm{~K}$ & $\partial T / \partial n=0$ \\
\hline & Step 1. & & & \\
$v$ & $15-25-37$ \\
$-50-70$ SLPM & 0 & 0 & $\partial v / \partial n=0$ \\
\hline \multirow{2}{*}{$I$} & $\partial I / \partial n=0$ & $\begin{array}{c}\text { Step 2. } \\
100-150-200 \\
-250-300 \mathrm{~A}\end{array}$ & $\partial I / \partial n=0$ & $\partial I / \partial n=0$ \\
\hline$A$ & $\partial A / \partial n=0$ & $\partial A / \partial n=0$ & $\partial A / \partial n=0$ & $\partial A / \partial n=0$
\end{tabular}

The torch was performed as three-dimensional and turbulent flow. Time-independent solutions were made. Ansys fluent and magnetohydrodynamic (MHD) modules were used together to solve the interaction between the electromagnetic field and the gas flow in the created geometry, and the results were obtained by changing the current value, mass flow rate, and geometry. Argon is used as the plasma gas. Values such as thermal conductivity, enthalpy, viscosity, electrical conductivity are taken from the sources [15-17] to simulate fully the thermal and electrical behavior of the argon gas at high temperatures. Firstly, the current and mass flow rate changes on an original geometry are investigated. Later geometry changes were made. Thus extending the length of the plasma jet was targeted.

A line was drawn from tip of the cathode to end of the outlet in order to determine length of the plasma jet and the data from the 200 points on it was taken. Received data line is shown in Fig. 2a.

The effects of mass flow rate (Step 1), current value (Step 2) and geometry (Step 3) changes were investigated in order to move the plasma jet furthest away from the torch exit without losing its temperature and velocity.

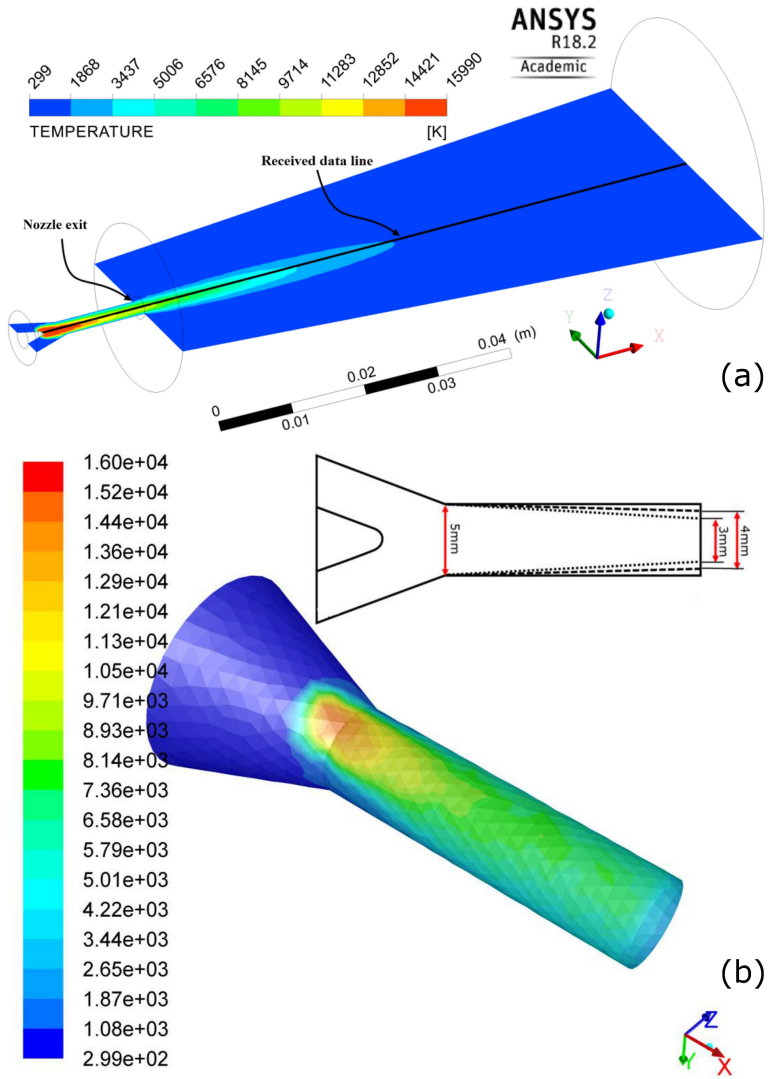

Fig. 2. Result of numerical analysis in parameter 300A37SLPM: (a) received data line, (b) arc-root attachment on nozzle and geometries of Step 3.

\section{Results and discussion}

In the first step, the effect of mass flow rate parameters of $15,25,37,50,70$ SLPM were investigated by keeping the current value constant at $200 \mathrm{~A}$. In Fig. 3, the temperature (Fig. 3a) and velocity (Fig. 3b) values taken at 40,60 , and $80 \mathrm{~mm}$ from the nozzle exit are plotted. When the graph was examined, it was observed that the exit temperature increased with increasing mass flow rate. For this reason, the increased mass flow rate is considered as an increase in the energy required to ionize. When the required energy is increased, the arc core diameter decreases and the arc length increases. As the arc length increases, higher energy is transferred to the nozzle exit due the smaller distance between the arc root and nozzle exit. This situation has been already shown in the literature $[4,6,11]$.

In the second step, the effect of different current values is investigated by keeping constant 37 SLPM parameters in which the mean temperature values are obtained in Fig. 3. Figure 4 shows the effect of the current value on the temperature (Fig. 4a) and velocity (Fig. 4b) values at 40,60 , and $80 \mathrm{~mm}$ from the torch output. As the current value increases, the temperature and velocity values tend to increase. For this reason, as the flow density increased at constant flow rate, the arc diameter increased and the arc length became longer. At $300 \mathrm{~A}$ current, the 

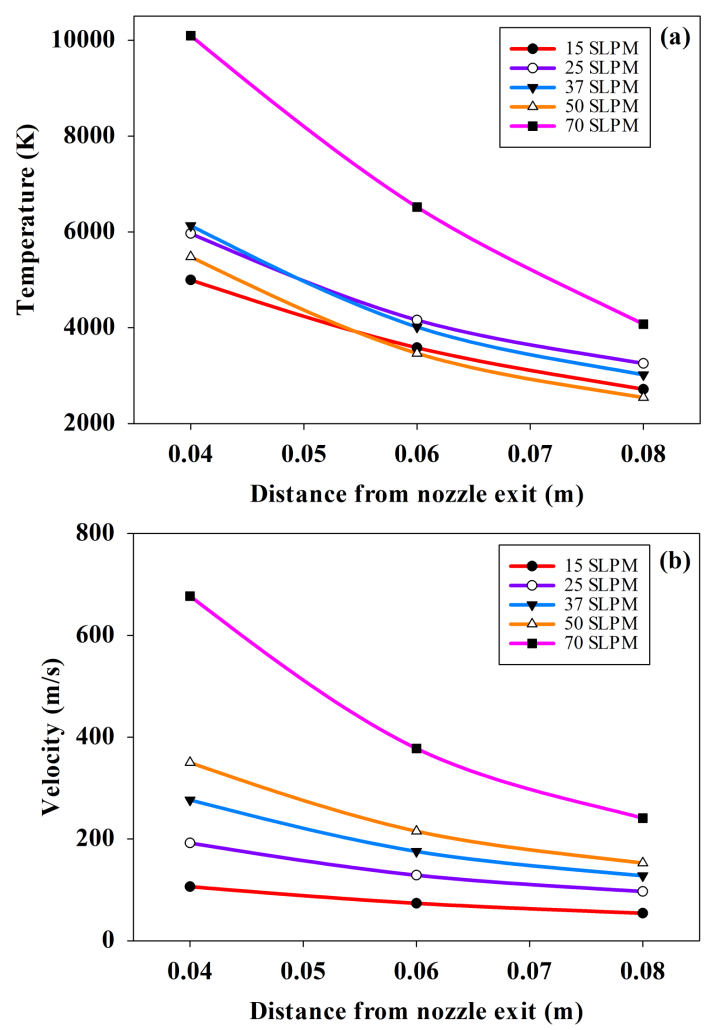

Fig. 3. Graphics at specific distances from nozzle exit depend on mass flow rate change: (a) temperature, (b) velocity.
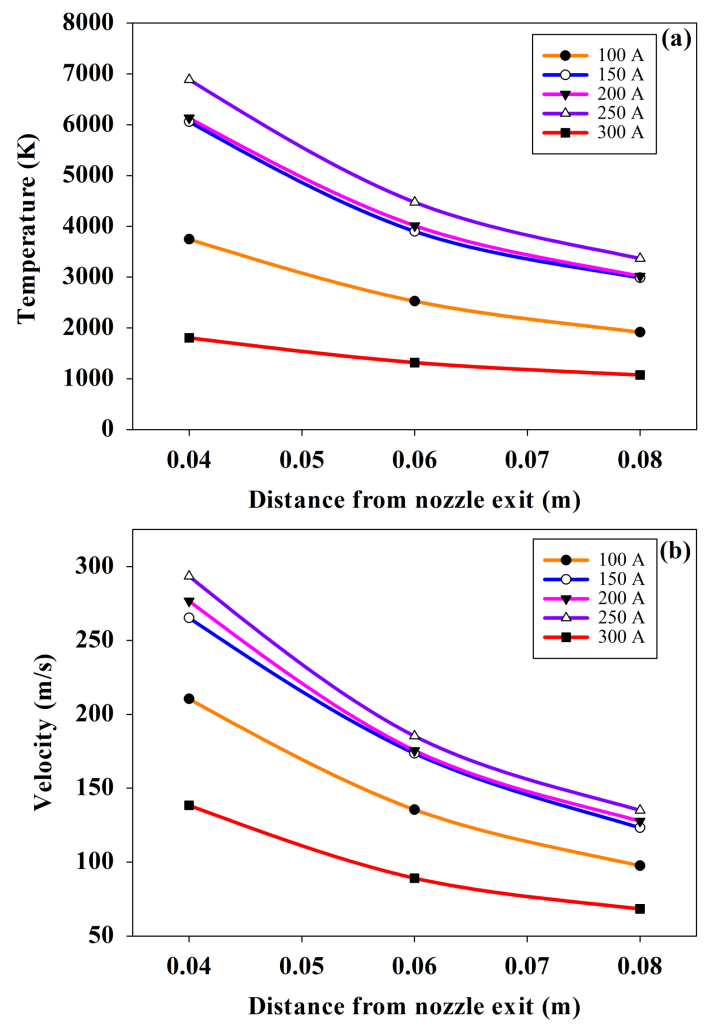

Fig. 4. Graphics at specific distances from nozzle exit depend on current value change: (a) temperature, (b) velocity. temperature and velocity at the nozzle exit reduced due to the shorter distance of the arc root position from the anode tip (Fig. 4a and b). As a result of this effect, the energy at the nozzle exit is lower if it is compared with longer arc root position. 150 and 200 A parameters are very close to each other. Compared with mass flow rate, the effect of current value seems to be comparatively less.

In the third step, the effect of geometry change is investigated by using the values of $250 \mathrm{~A}$ and 70SLPM in which the highest temperature and velocity values according to Figs. 3 and 4 . Geometry effects on the temperature and velocity of the plasma jet are shown in Fig. 5. The temperature (Fig. 5a) and velocity (Fig. $5 \mathrm{~b})$ values at certain distances are taken from the exit, and the graphs in Fig. 5 are drawn. As the exit diameter narrows, the temperature decreases and the velocity values increase. Thanks to the geometry change, temperature and velocity values have been moved further from the exit.
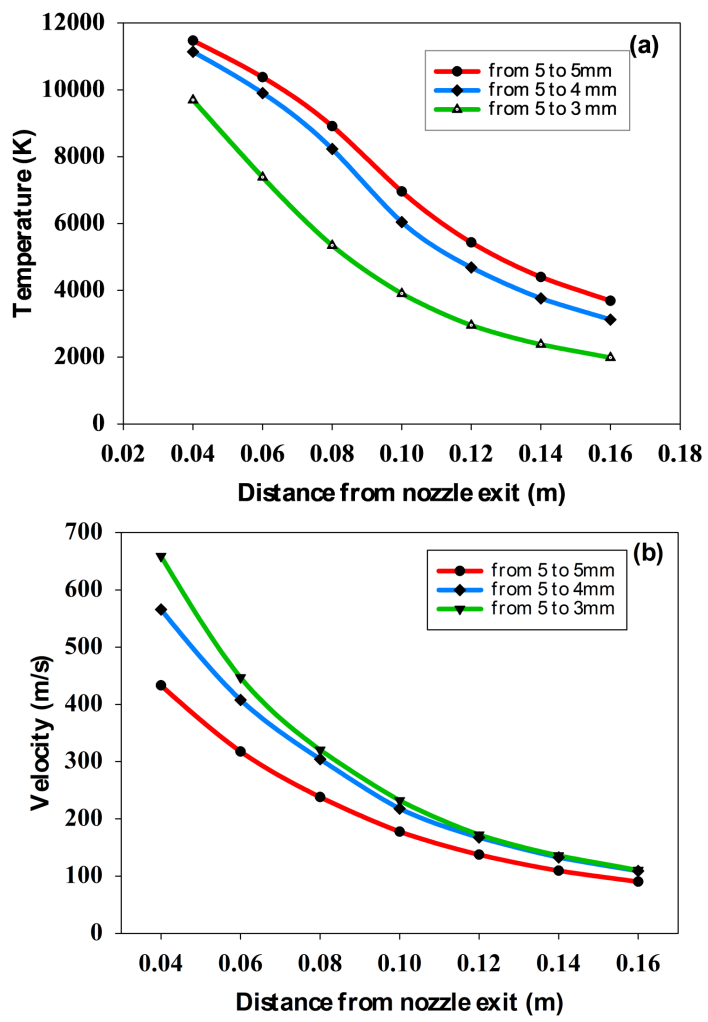

Fig. 5. Graphics at specific distances from nozzle exit depend on geometry change: (a) temperature, (b) velocity.

\section{Conclusion}

In this study, torch design and numerical analysis were done for plasma atomization method. The desired temperature to be obtained from the plasma torch is directly related to the arc length and arc position in the torch. Increased flow and current value increase the output temperature and velocity. However, high current values can 
reduce arc length contrary to expectations. As a result of this study, it is concluded that the desired plasma jet geometry for high velocity and temperature at the outside the torch could be achieved by making appropriate modifications to the geometry.

\section{Acknowledgments}

This research has been supported by the Scientific and Technological Research Council of Turkey (TÜBİTAK). Project Number: 215M895.

\section{References}

[1] J.M. Bauchire, J.J. Gonzalez, A. Gleizes, Plasma Chem. Plasma Process. 17, 409 (1997).

[2] R. Westhoff, J. Szekely, J. Appl. Phys. 70, 3455 (1991).

[3] S. Paik, P. C. Huang, J. Heberlein, E. Pfender, Plasma Chem. Plasma Process. 13, 379 (1993).

[4] Z. Guo, S. Yin, H. Liao, S. Gu, Int. J. Heat Mass Transf. 80, 644 (2015).

[5] J.J. Gonzalez, P. Freton, A. Gleizes, J. Phys. D Appl. Phys. 35, 3181 (2002).

[6] L. He-Ping, E. Pfender, X. Chen, J. Phys. D: Appl. Phys., 36(9), 1084, (2003).
[7] J. Park, J. Heberlein, E. Pfender, G. Candler, C.H. Chang, Plasma Chem. Plasma Process. 28, 213 (2008).

[8] K. Ramachandran, J.L. Marqués, R. Vaßen, D. Stöver, J. Phys. D Appl. Phys. 39, 3323 (2006).

[9] P. Eichert, M. Imbert, C. Coddet, J. Thermal Spray Tech. 7, 505 (1998).

[10] E. Meillot, D. Guenadou, C. Bourgeois, Plasma Chem. Plasma Process. 28, 69 (2008).

[11] B. Selvan, K. Ramachandran, K.P. Sreekumar, T.K. Thiyagarajan, P.V. Ananthapadmanabhan, Vacuum 84, 444 (2009).

[12] J.P. Trelles, E. Pfender, J. Heberlein, Plasma Chem. Plasma Process. 26, 557 (2006).

[13] A.B. Murphy, P. Kovitya, J. Appl. Phys. 73, 4759 (1993).

[14] R. Huang, H. Fukanuma, Y. Uesugi, Y. Tanaka, IEEE Trans. Plasma Sci. 39, 1974 (2011).

[15] D.L. Evans, R.S. Tankin, Phys. Fluids 10, 1137 (1967).

[16] A.B. Murphy, C.J. Arundelli, Plasma Chem. Plasma Process. 14, 451 (1994).

[17] M.I. Boulos, P. Fauchais, E. Pfender, Thermal Plasmas: Fundamentals and Applications, Springer Sci. \& Business Media, New York 1994. 